\title{
Phenylalanine Hydroxylase (PAH) Genotyping in PKU Argentine Patients
}

Journal of Inborn Errors

of Metabolism \& Screening 2019, Volume 7: e20190012

DOI: 10.1590/2326-4594-JIEMS-2019-0012

\author{
Rosa E. Enacán', Mariana Nuñez Miñana², Luis Fernandez ${ }^{3}$, \\ Maria Gabriela Valle', Mercedes Salerno', Claudia I. Fraga', \\ Fernando Santos-Simarro ${ }^{3}$, Laura Prieto', Pablo Lapunzina ${ }^{3}$, \\ Norma Specola ${ }^{2}$, Ana Elena Chiesa ${ }^{1}$
}

\begin{abstract}
Phenylketonuria (PKU, OMIM 261600) is predominantly caused by mutations in the PAH gene. One hundred and three Argentine PKU patients were studied by Sanger sequencing; 101 were completely characterized ( $90.3 \%$ were compound heterozygotes). Fifty-four different pathogenic variants were identified. Mutations were distributed all along the PAH gene but concentrated in exon 7 (26\%), 12 (12\%), 11 (10\%), and 6 (10\%). 77\% were missense, and 77\% affected the enzyme catalytic domain, nine mutations accounted for $57 \%$ of 179 studied alleles: p.Arg261Gln (Allele frequency(AF):10.6\%), c.1066-11G>A (AF:9,5\%), p.Arg408Trp (AF:8,3\%), p.Tyr414Cys (AF:5,5\%), p.Ala403Val, p.Val388Met, and p.Arg158Gln (AF: 5\% each), p.Leu48Ser, and p.lle65Thr (AF:4\% each). The predicted phenotype was assigned by Guldberg's arbitrary value (AV) and compared with the clinical phenotype based in tolerance to Phe intake. 29.1\% (n:30) were hyperphenylalaninemias, 18.5\% (n:19) mild-PKU, 27.2\% (n:28) moderate-PKU and 25.2 \% (n:26) classical-PKU. Genotype/phenotype correlation was statistically significant ( $p<0.001)$ Overall concordance was $62,5 \%$ : $93.3 \%$ in hyperphenylalaninemia, $64.7 \%$ in mild-PKU and $65.4 \%$ in classical patients. The moderate-PKU showed a weak concordance (17\%) with milder AV prediction than clinical assessment. 74\% of discordant moderate patients harbored p.Arg261Gln, and p.Val388Met. Our cohort is highly heterogeneous, with predominant Mediterranean influence (mainly Spanish), but with differences with other Latin-American countries.
\end{abstract}

\section{Keywords}

$\mathrm{Pku}$, genotype, phenylalanine hydroxylase.

\section{Introduction}

Phenylketonuria (PKU, OMIM 261600) is an inborn error of metabolism of phenylalanine (Phe), predominantly caused by mutations in the phenylalanine hydroxylase $(P A H)$ gene located on chromosome 12 (12q22-q24.2) [1]

To date, more than 1100 variants have been reported in $P A H$, and new data are continuously added to databases (http:// www.biopku.org/home/pah.asp) $P A H$ pathogenic variants lead to impaired function of the hepatic enzyme which catalyzes the conversion of the essential amino acid L-phenylalanine (Phe) to L-tyrosine (Tyr), a precursor of the neurotransmitters dopamine, noradrenaline, and adrenaline.

Although the exact pathogenesis of the disease remains unclear, the defect causes Phenylalanine (Phe) accumulation (hyperphenylalaninemia) and neurotransmitter depletion and, unless treated opportunely, patients suffer from intellectual impairment of different magnitude. The disease is inherited as an autosomal recessive disorder, and its severity is a spectrum that reflects in the phenylalanine blood levels the residual activity of the affected enzyme. The majority of reported patients are

\footnotetext{
${ }^{1}$ Fundación de Endocrinología Infantil (FEI). Buenos Aires, Argentina

2 Unidad de Metabolismo. Hospital de Niños "Sor Ludovica" de La Plata, Buenos Aires, Argentina

${ }^{3}$ Instituto de Genética Médica y Molecular (INGEMM), Hospital Universitario La Paz, IdiPAZ, Universidad Autónoma de Madrid; CIBERER, Centro de Investigación Biomédica en Red de Enfermedades Raras, ISCIII, Madrid, España
}

Received September 23, 2019, and in revised form November 04, 2019. Accepted for publication November 13, 2019.

\section{Corresponding Author:}

Ana Elena Chiesa, Fundación de Endocrinología Infantil (FEI). Buenos Aires, Argentina

Email: achiesa@cedie.org.ar 
compound heterozygotes, with high heterogeneity. Genotype varies in different populations, but ancestral origin can be followed deriving from regions of Europe and Asia.[2,3] Also, biochemical and clinical phenotypes are highly variable, suggesting the influence of other modifier genes, possibly those responsible for amino acid brain barrier transporters. Consequently, it is often difficult to ascertain the phenotypic consequences of a pathogenic variant. $[4,5]$

In the last decades, new treatments became suitable for some PKU patients.[6,7] Some of their therapeutic effects are based on rescuing the residual activity of the affected enzyme.[8,9] In this context, PKU genotyping provides valuable information and available increasing data on databases represents a useful resource for the clinical management of the disease. Thus, the report of PKU cohorts helps to clarify the phenothype-genotype relationships and allows the individualized follow up of affected patients and their selection for new treatment options. $[3,10,11]$

Newborn screening for PKU was established in Argentina in 1985 and developed in a decentralized model based on phenylalanine determinations in DBS at maternity discharge with confirmation with Phe, tyrosine levels, and Phe/Tyr ratio.

Since then, among the settled programs, the ones of Buenos Aires Province, and FEI (Fundación de Endocrinología Infantil) (a non-profit private institution ) found in $>6.000 .000$ births and incidence of PKU and HPA of 1:13000. [12] The detected patients are followed in two specialized centers.

\section{Aim}

In this study, we analyzed for the first time the genotype of a large cohort of Argentine PKU patients detected and followed up by both centers to identify the PAH mutation spectrum prevalent in our country and describe the phenotype-genotype associations.

\section{Patients and Methods}

Patients were recruited among the 420 patients with hyperphenylalaninemia followed up either in the metabolic unit of the Sor Maria Ludovica Children's hospital (La Plata, Buenos Aires Province, Argentina) or in the PKU clinic of FEI (Fundación de Endocrinología Infantil) (City of Buenos Aires). Their diagnosis was based on the determination of phenylalanine levels $>120 \mathrm{ug} / \mathrm{dl}$ and Phe/Tyr ratio.

Genotype was carried out in 103 patients (52 female, 51 males) from 90 unrelated families. Thirteen of them had more than one affected individual (12 pairs of brothers and a mother and child born from a blood relationship). To assess phenotype, patients were classified according to their Phe tolerance at age 4-7 years, following Guldberg's phenotype classification as classic PKU, moderate PKU, mild PKU, or Hyperphenylalaninemia.[13]

Genomic DNA was obtained from peripheral blood lymphocytes using Puregene DNA Purification Kit (QIAGEN). Coding exons and exon-intron boundaries of $P A H$
(NM_000277.1) were PCR-amplified and analyzed by Sanger sequencing. Mutation nomenclature follows the HGVS guidelines (http://www.HGVS.org/varnomen). Once genotyped, the arbitrary value (AV) by Guldberg's was assigned to each known mutation obtained from BIOPKU database and phenotype was predicted accordingly. Phenotypes resulting from a combination of the two mutant alleles were expressed as the sum of the two AVs (SUM AVs).[13] The correlation between tolerance and predicted phenotype was calculated with Spearman correlation (significance p: $<0.005$ ). Concordance between the predicted and the observed phenotype for patients with complete mutation analysis was also calculated for the whole group and every phenotype category.

Aditionally, the recently communicated genotypic phenotype value (GPVs) for each patient was asigned with the APV scores provided for each mutation in http://www.biopku.org/home/ pah.asp following Garbade et al algorithm.[14]

The allelic frequency was calculated in 179 identified affected alleles, calculating only one patient in each pair of siblings that shared two common alleles and three alleles in the discordant couple (sharing only one allele).

The study was approved by the Human Ethics Committee of the Buenos Aires Children's Hospital. Informed written consent was obtained from parents and assent from children older than seven years.

\section{Results}

\section{Phenotype}

According to Guldberg's classification 26 (25.2\%) patients had classical PKU, 28 (27.2\%) moderate PKU, 19 (18.5\%) mild PKU and 30 (29.1\%) hyperphenylalaninemia (HPA).

\section{Genotype: PAH mutation spectrum}

One hundred and one out of 103 patients were characterized entirely, and in 2, only one mutation was found. Fifty four different pathogenic mutations, including three previously unreported variations and one intron duplication spanning an entire exon that needs further characterization, were identified in 179 out of the181 independent alleles. Ninety-one patients (90.3\%) were compound heterozygotes.

Mutations were distributed all along the PAH gene being $14 \%$ intronic and the others concentrated in exon $7(26 \%)$, exon $12(12 \%)$, exon $11(10 \%)$, exon $6(10 \%)$ and exons 10,2 and $3(6 \%$ each). Seventy seven percent were missense, $12 \%$ nonsense, $11 \%$ frameshift. 77\% were placed in the enzyme catalytic domain, $16 \%$ in the regulatory, and $7 \%$ in the oligomerization zone. Twelve pairs of siblings that had a similar phenotype shared the same two pathogenic mutations, while the one with discordant phenotype shared only one allele (p.Leu348Val), being the second p.Arg261Gln for one sibling, that was classified as moderate PKU, and p.Val245Ala for his brother that had a persistent HPA. 
Their mother was not initially suspected of having PKU, but Phe levels revealed slight hyperphenylalaninemia.

Nine mutations accounted for $57 \%$ of alleles screened: p.Arg261Gln (Allele frequency (AF):10.6\%), c.1066-11G>A (AF:9.5\%), p.Arg408Trp (AF:8.3\%), p.Tyr414Cys (AF:5.5\%), p.Ala403Val (AF:5\%), p.Val388Met (AF:5\%), p.Arg158Gln (AF:5\%), p.Leu48Ser (AF:4\%) and p.Ile65Thr (4\%).

Table 1 shows information on genotype, calculated SUM AVs for both alleles, phe tolerance mg/day, phenotype assessed clinically and classified by Guldberg's, concordance with predicted genotype (Yes/No), GPVs and results of the $48 \mathrm{hs}$ $\mathrm{BH}_{4}$ test when performed, considering as a positive response a decrease $\geq 30 \%$ of the Phe basal levels under a stable Phe consumption.[15]

The p.Arg261Gln was the most frequent mutation in our cohort, affecting 18 (17.4\%) of our patients.Two patients were homozygous with classic and moderate phenotypes. The other 16 were heterozygous and showed phenotypes that covered the whole clinical spectrum, depending on the second mutation found. (six HPA, two mild, eight moderates). AVs values ranged from 5 to 12 . Response to $\mathrm{BH}_{4}$ test was positive in the 12 patients tested, including the homozygous.

The c.1066-11G>A variant affected 16 patients (15.5\%). Their phenotype was classic in the two homozygous, and nine heterozygous, mild in 4 and HPA in 1. Response to BH4 was positive in 4 out of 6 patient tested.

The p. Arg408Trp was present 16 patients (15.5\%). Four were siblings, homozygous, and had the classical phenotype. In the remaining patients, 4 showed classical, 5 moderate and 5 the HPA phenotype, depending on the other mutation found. Response to $\mathrm{BH}_{4}$ was positive when combined with mutations with higher AVs. Three of these patients were tested, and two responded to $\mathrm{BH}_{4}$.

The p.Tyr414Cys change was present in 11 patients (10.6\%). All were heterozygous with phenotype going from classical to moderate when associated with pArg408Trp or p.Val388Met and developing HPA in the two patients that associated p.Asp415Asn. $\mathrm{BH}_{4}$ response was present in 4 out of 6 patients tested.

The p.Ala403Val, p.Val388Met, and p.Arg158Gln variants accounted for $10(9.7 \%), 9(8.7 \%)$ and $8(7.7 \%)$ patients respectively. While p.Ala403Val was present always in mild forms patients with p.Val388Met, and p.Arg158Gln had more restricted tolerances ranging from classical to mild forms. While our patients with p.Ala403Val did not need to try $\mathrm{BH} 4$, responses in those carrying the other two mutations were variable and related to the allelic combination.

Finally the p.Leu48Ser, and p.Ile65Thr changes were present in $8(7.7 \%)$, and $7(6,8 \%)$ patients respectively. While the first showed a milder clinical spectrum, the second included the whole range of clinical features depending on the other present mutation. Accordingly, responses to $\mathrm{BH}_{4}$ were variable.

\section{Phenotype-genotype correlation}

In 96 patients, the predicted phenotype assigned by Guldberg's activity value for both alleles (SUM AVs) was compared with the observed phenotype. Overall correlation with predicted phenotype was significant ( $.0 .77 ; \mathrm{p}<0.001)$ with overall concordance between prediction and clinical assessment of $62,5 \%$. This concordance was $93 \%$ for HPA, 65\% for mild PKU, and $65 \%$ for classical PKU patients. On the contrary, a weak concordance (17\%) was found in the moderate group where predicted empiric phenotype was milder than the one assessed by

Table 1. Genotype (cDNA and protein), SUM AVs, Phe tolerance(mg/day), Phenotype, concordance (C), GPVs and BH4 response in the 103 studied patients

\begin{tabular}{|c|c|c|c|c|c|c|c|c|c|c|}
\hline \multirow[b]{2}{*}{1} & \multicolumn{2}{|c|}{$\begin{array}{c}\text { Allele } 1 \\
\text { cDNA Protein }\end{array}$} & \multicolumn{2}{|c|}{$\begin{array}{c}\text { Allele2 } \\
\text { cDNA Protein }\end{array}$} & \multirow{2}{*}{$\begin{array}{c}\text { SUM } \\
\text { AVs } \\
8\end{array}$} & \multirow{2}{*}{$\begin{array}{c}\text { Phe } \\
\text { Tolerance } \\
\text { mg/day }\end{array}$} & \multirow{2}{*}{$\begin{array}{l}\text { Phenotype } \\
\text { Moderate }\end{array}$} & \multirow{2}{*}{$\begin{array}{l}\text { (C) } \\
\text { no }\end{array}$} & \multirow{2}{*}{$\begin{array}{c}\text { GPVs } \\
1.6\end{array}$} & \multirow{2}{*}{$\begin{array}{c}\mathrm{BH} 4 \\
\text { response } \\
\text { yes }\end{array}$} \\
\hline & c. $782 \mathrm{G}>\mathrm{A}$ & p.Arg261Gln & c. $782 \mathrm{G}>\mathrm{A}$ & p.Arg261Gln & & & & & & \\
\hline 2 & c.782G $>A$ & p.Arg261Gln & c. $782 \mathrm{G}>\mathrm{A}$ & p.Arg261Gln & 8 & 300 & Classical & no & 1.6 & nt \\
\hline 3 & c.782G $>A$ & p.Arg261Gln & c. $1208 C>T$ & p.Ala403Val & 12 & 1750 & HPA & yes & 9.8 & nt \\
\hline 4 & c. $782 \mathrm{G}>\mathrm{A}$ & p.Arg261Gln & c. $1208 C>T$ & p.Ala403Val & 12 & 1600 & HPA & yes & 9.8 & $\mathrm{nt}$ \\
\hline 5 & c. $824 C>G$ & p.Pro275Arg & c. $782 \mathrm{G}>\mathrm{A}$ & p.Arg261Gln & 9 & 900 & HPA & yes & 5 & yes \\
\hline 6 & $c .824 C>G$ & p.Pro275Arg & c. $782 \mathrm{G}>\mathrm{A}$ & p.Arg261Gln & 9 & 900 & HPA & yes & 5 & yes \\
\hline 7 & c.782G $>A$ & p.Arg261Gln & c. $204 \mathrm{~A}>\mathrm{T}$ & p.Arg68Ser & 8 & 1000 & HPA & yes & 5.4 & nt \\
\hline 8 & c. $782 \mathrm{G}>\mathrm{A}$ & p.Arg261Gln & c. $1223 \mathrm{G}>\mathrm{A}$ & p.Arg408Gln & 8 & 900 & HPA & yes & 5.2 & yes \\
\hline 9 & c. $782 \mathrm{G}>\mathrm{A}$ & p.Arg261Gln & c. $1222 \mathrm{C}>\mathrm{T}$ & p.Arg408Trp & 5 & 360 & Moderate & no & 5.2 & no \\
\hline 10 & c. $782 \mathrm{G}>\mathrm{A}$ & p.Arg261Gln & c. $1042 C>G$ & p.Leu348Val & 8 & 580 & Mild & yes & 1,5 & yes \\
\hline 11 & c. $782 \mathrm{G}>\mathrm{A}$ & p.Arg261Gln & $c .838 G>A$ & p.Glu280Lys & 5 & 500 & Mild & yes & 1.6 & yes \\
\hline 12 & c.782G $>A$ & p.Arg261Gln & c.194T>C & p.lle65Thr & 8 & 360 & Moderate & no & 1.6 & yes \\
\hline 13 & c. $782 \mathrm{G}>\mathrm{A}$ & p.Arg261Gln & c. $728 \mathrm{G}>\mathrm{A}$ & p.Arg243Gln & 5 & 360 & Moderate & no & 1.6 & yes \\
\hline 14 & c. $782 \mathrm{G}>\mathrm{A}$ & p.Arg261Gln & $c .842+3 G>C$ & & 5 & 350 & Moderate & no & 1.6 & yes \\
\hline
\end{tabular}


Table 1. Cont.

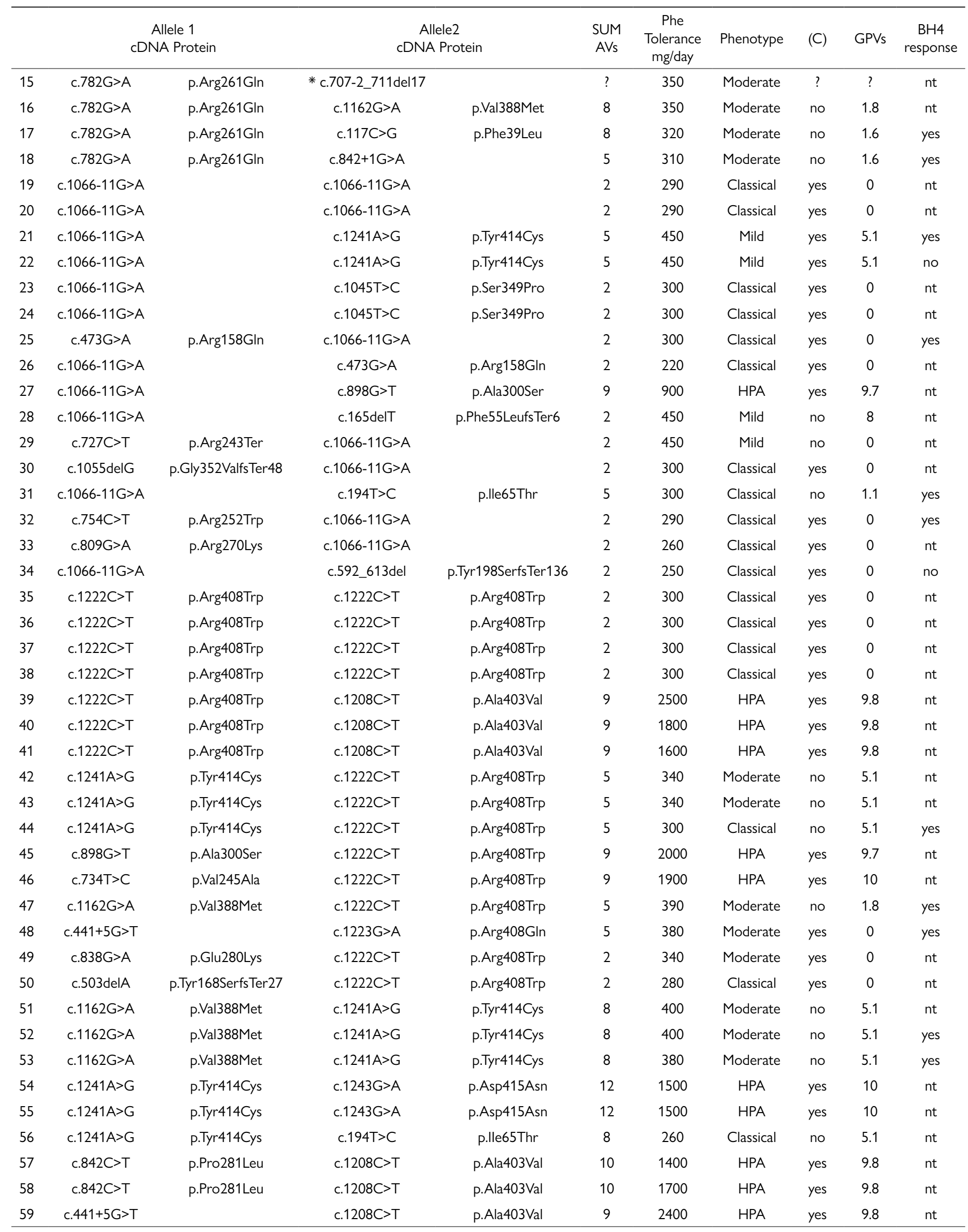


Table 1. Cont.

\begin{tabular}{|c|c|c|c|c|c|c|c|c|c|c|}
\hline \multirow[b]{2}{*}{60} & \multicolumn{2}{|c|}{$\begin{array}{c}\text { Allele } 1 \\
\text { cDNA Protein }\end{array}$} & \multicolumn{2}{|c|}{$\begin{array}{c}\text { Allele2 } \\
\text { cDNA Protein }\end{array}$} & \multirow{2}{*}{$\begin{array}{c}\text { SUM } \\
\text { AVs } \\
12\end{array}$} & \multirow{2}{*}{$\begin{array}{c}\begin{array}{c}\text { Phe } \\
\text { Tolerance } \\
\text { mg/day }\end{array} \\
1500\end{array}$} & \multirow{2}{*}{$\begin{array}{c}\text { Phenotype } \\
\text { HPA }\end{array}$} & \multirow{2}{*}{$\begin{array}{l}\text { (C) } \\
\text { yes }\end{array}$} & \multirow{2}{*}{$\begin{array}{r}\text { GPVs } \\
9.8\end{array}$} & \multirow{2}{*}{$\begin{array}{c}\begin{array}{c}\mathrm{BH} 4 \\
\text { response }\end{array} \\
\mathrm{nt}\end{array}$} \\
\hline & c. $829 \mathrm{~T}>\mathrm{G}$ & p.Tyr277Asp & c. $1208 C>T$ & p.Ala403Val & & & & & & \\
\hline 61 & c. $1208 \mathrm{C}>\mathrm{T}$ & p.Ala403Val & c. $194 \mathrm{~T}>\mathrm{C}$ & p.lle65Thr & 12 & 1500 & HPA & yes & 9.8 & $\mathrm{nt}$ \\
\hline 62 & c. $1162 \mathrm{G}>\mathrm{A}$ & p.Val388Met & c. $143 T>C$ & p.Leu48Ser & 8 & 600 & Mild & yes & 2 & $\mathrm{nt}$ \\
\hline 63 & c.781C>T & p.Arg261Ter & c. $1162 \mathrm{G}>\mathrm{A}$ & p.Val388Met & 5 & 550 & Mild & yes & 1.8 & yes \\
\hline 65 & c. $473 G>A$ & p.Arg158Gln & c. $1162 \mathrm{G}>\mathrm{A}$ & p.Val388Met & 8 & 290 & Classical & no & 1.8 & $\mathrm{nt}$ \\
\hline 66 & c. $473 \mathrm{G}>\mathrm{A}$ & p.Arg158Gln & c. $473 G>A$ & p.Arg158Gln & 2 & 320 & Moderate & yes & 0 & $\mathrm{nt}$ \\
\hline 67 & c. $473 \mathrm{G}>\mathrm{A}$ & p.Arg158Gln & c. $1169 A>G$ & p.Glu390Gly & 9 & 1500 & HPA & yes & 7 & $\mathrm{nt}$ \\
\hline 68 & c. $473 \mathrm{G}>\mathrm{A}$ & p.Arg158Gln & c. $194 \mathrm{~T}>\mathrm{C}$ & p.lle65Thr & 5 & 500 & Mild & yes & 1.1 & no \\
\hline 69 & c. $473 \mathrm{G}>\mathrm{A}$ & p.Arg158GIn & Not found & & & 330 & Moderate & & & yes \\
\hline 73 & $c .527 \mathrm{G}>\mathrm{T}$ & p.Arg176Leu & c. $143 T>C$ & p.Leu48Ser & 12 & 2500 & HPA & yes & 9.7 & $\mathrm{nt}$ \\
\hline 74 & c. $143 \mathrm{~T}>\mathrm{C}$ & p.Leu48Ser & c. $1045 T>C$ & p.Ser349Pro & 5 & 450 & Mild & yes & 2 & nt \\
\hline 75 & c. $143 T>C$ & p.Leu48Ser & c. $1045 T>C$ & p.Ser349Pro & 5 & 450 & Mild & yes & 2 & yes \\
\hline 76 & c. $809 \mathrm{G}>\mathrm{A}$ & p.Arg270Lys & c. $143 T>C$ & p.Leu48Ser & 5 & 400 & Moderate & no & 2 & no \\
\hline 77 & c. $143 T>C$ & p.Leu48Ser & c. $204 \mathrm{~A}>\mathrm{T}$ & p.Arg68Ser & 8 & 500 & Mild & yes & 5.4 & $\mathrm{nt}$ \\
\hline 78 & $c .781 C>T$ & p.Arg261Ter & c. $1184 C>G$ & p.Ala395Gly & 5 & 2000 & HPA & no & 5 & $\mathrm{nt}$ \\
\hline 79 & c. $781 \mathrm{C}>\mathrm{T}$ & p.Arg261Ter & c. $1184 C>G$ & p.Ala395Gly & 5 & 1500 & HPA & no & 5 & yes \\
\hline 80 & c. $1315+1 G>A$ & & c. $916 A>G$ & p.lle306Val & 9 & 1300 & HPA & yes & 9.8 & $\mathrm{nt}$ \\
\hline 81 & c. $1315+1 G>A$ & & c. $916 A>G$ & p.lle306Val & 9 & 1300 & HPA & yes & 9.8 & $\mathrm{nt}$ \\
\hline 88 & c.781C>T & p.Arg261Ter & c. $612 T>G$ & p.Tyr204Ter & 2 & 250 & Classical & yes & 0 & $\mathrm{nt}$ \\
\hline 89 & c.47_48delCT & p.Ser16Ter & c.183_187delCCTGA & p.Asn61AsnfsTer4 & $?$ & 320 & Moderate & $?$ & 0 & no \\
\hline 90 & c. $526 \mathrm{C}>\mathrm{T}$ & p.Arg176Ter & c. $842 C>T$ & p.Pro281Leu & 3 & 300 & Classical & no & 0 & $\mathrm{nt}$ \\
\hline 91 & c.745C>T & p.Leu249Phe & Not found & & $?$ & 380 & Moderate & $?$ & $?$ & $\mathrm{nt}$ \\
\hline 92 & Dup 15 /del E6 & & c.847A>T & p.lle283Phe & $?$ & 390 & Moderate & $?$ & 0 & $\mathrm{nt}$ \\
\hline 93 & c. $441+5 G>T$ & & c. $1045 T>C$ & p.Ser349prol & 2 & 600 & Mild & no & 0 & $\mathrm{nt}$ \\
\hline 94 & $c .727 \mathrm{C}>\mathrm{T}$ & p.Arg243Ter & c.194T>C & p.lle65Thr & 5 & 290 & Classical & no & 1.1 & yes \\
\hline 95 & c.734T >C & p.Val245Ala & c. $1042 C>G$ & p.Leu348Val & 12 & 1400 & HPA & yes & 10 & $\mathrm{nt}$ \\
\hline 96 & c. $1199+17 G>A$ & & c. $168+19 T>C$ & & 9 & 900 & HPA & yes & $?$ & yes \\
\hline 97 & c. $1157 A>G$ & p.Tyr386Cys & c. $1169 A>G$ & p.Glu390Gly & 9 & 1000 & HPA & yes & 7 & yes \\
\hline 98 & c. $162 \mathrm{~A}>\mathrm{T}$ & ${ }^{*}$ p.Leu54Phe & c. $1042 C>G$ & p.Leu348Val & $?$ & 500 & Mild & $?$ & $?$ & nt \\
\hline 99 & c.451delG & *p.Asp151llefsTer44 & c. $1249 \mathrm{~T}>\mathrm{A}$ & p.Tyr417Asn & $?$ & 500 & Mild & $?$ & $?$ & no \\
\hline 100 & c. $1169 A>G$ & p.Glu390Gly & c.1089delG & p.Lys363Asnfs & 9 & 440 & Mild & no & 7 & yes \\
\hline 101 & c. $665 \mathrm{~A}>\mathrm{G}$ & p.Asp222Gly & $c .727 C>T$ & p.Arg243Ter & 5 & 400 & Moderate & no & 6 & $\mathrm{nt}$ \\
\hline 102 & $c .824 C>G$ & p.Pro275Arg & c. $441+5 G>T$ & & 6 & 340 & Moderate & no & 5 & nt \\
\hline 103 & c. $809 \mathrm{G}>\mathrm{A}$ & p.Arg270Lys & $c .833 C>A$ & p.Thr278Asn & 2 & 330 & Moderate & YES & 0 & yes \\
\hline
\end{tabular}

nt: Not tested. ?: Unknown. *: not previously described 
tolerance. This happened in 19/23 patients, 14 of which harbored p.Arg $261 \mathrm{Gln}$ or p.Val388Met mutations and represented $74 \%$ of discordant moderate PKU patients .

\section{Discussion}

In this study, we describe for the first time in Argentina, a large number of $P A H$ pathogenic variants in a cohort of PKU patients to characterize their genotype and phenotype. As molecular testing is not routinely included in Argentina neither in the NBS programs nor in the follow-up, these are the first results of a large cohort of PKU patients studied.

Argentina is an extense country with a complex and varied immigration pattern.Our cohort represents the first study on unselected patients detected through neonatal screening regardless of their residence. So, many of the patients are from Buenos Aires City and Province, and some of them came from the rest of the country where the colonization may differ. Due to this situation, some population subsets included may vary depending on the studied area: e.g. the area of Buenos Aires due to the river access to the country by different European colonizers was more influenced by immigration than inner regions of the country. Unfortunately, genealogical data were not retrieved.

We identified in 103 patients, 54 different PAH mutations with $90.5 \%$ of compound heterozygous. Accordingly, PKU phenotypes in our patients were highly heterogeneous and covered all the spectrum of PAH activity with a distribution that shifts to a milder pattern than the one communicated for central Europe and resembling the Mediterranean ethnic composition. $[16,17]$ Despite heterogeneity, the genotype-phenotype correlation found was strong and would have allowed in $2 / 3$ of the patients the precise prediction of the clinical form. Exon 7, 12, and 11 were the more affected sites $(26 \%, 12 \%$, and $11 \%$ respectively).

In agreement with Desviat and coworkers our population resembles the genetic diversity of PAH deficiency in the Spanish population with a predominance of $c .1066-11 \mathrm{G}>\mathrm{A}$, a frequently reported PKU mutation in PAHdb affecting Mediterranean patients.[17] The variants p.Ala403Val, p.Ile65Thr, and p.Val388Met found in the Spanish population were also present in ours explaining a milder phenotype.

The p.Arg261Gln variant, prevalent in Italian, Spanish and Portuguese PKU, was the most prevalent in our cohort pointing out the predominant influence of these immigration waves. $[17,18,19]$ Nevertheless, p.Arg408Trp, prevalent in central and northern Europe was also common in our patients leading to all the phenotype spectrum with severe classic forms when homozygous.[16]

Despite the shared history of Spanish colonization, our findings differ with other Latinamerican published data on PKU genotypes. In a recent Chilean report, Hamilton et al. also described a high heterogeneity and the presence of the already known prevalent p.Val388Met, c.442-?_509+?del and c.1066-11G $>A$ in their patients. [20,21] Also, Santos et al. in Southeastern Brasil communicated the prevalence of p.Val388Met, p Arg261Gln,
IVS10-11G $>$ A and p. Ile65Thr. [19] So, our coinciding findings underscore the influence of the Iberian Peninsula migration to our continent. Nevertheless, the proportion of affected alleles was different in our study and p.Arg261Gln was nor so frequent in their cohorts . Similarly, p.Val388Met reported by Brasil, Mexico and Chile as prevalent $(21.2 \%, 8,3 \%$, and $17,3 \%)$ accounted for only $5 \%$ of our patients.[19,21,22]

The phenotype was also heterogeneous in our patients ranging from HPA to classical PKU, probably due to allelic complementation. Thus, p.ArgA261Gln assigned with intermediate levels of activity showed different behavior depending on the other present allele but keeping residual activity when homozygous.Similarly p.Arg408Trp, although proven with no residual activity, was present all through the phenotype spectrum of our cohort with classical forms in homozygous and milder forms in patients carrying less deleterious mutations in the other allele.

Regarding p.Ala403Val, p.Arg158Gln, p.Leu48Ser, p.Ileu65T and, pTyr414Cys, these changes kept their enzyme activities that were reflected, as described, in the phenotype.[23]

Substantial evidence is available on the utility of genotypephenotype correlation. [24,25,26]

Although European consensus points out the arbitrarity of phenotype classification[27], a relatively good correlation with predicted phenotype was found in our cohort.

As observed by others, discordant predictions were found mainly in the moderate group or the border of categories assigned. $[21,23]$ Intrinsic conformational changes in the mutated enzymes driven by interallelic interaction may explain to some degree the inaccurate prediction in this group of patients $[28,29,30]$.

As Hamilton et al. in Chile, some mutations found in our cohort showed a weak correlation with the expected phenotype. [21] Thus, the p.Arg261Gln, and p.Val388Met changes were present in two-thirds of our moderate PKU discordant patients. These variants are described with residual activity but affecting the folding and the active tetramer formation of the enzyme $[3,30]$.

Another issue that may affect the concordance estimation is the accurate assessment of Phe intake allowance (Phe tolerance). True Phe tolerance in PKU patients is not so easy to evaluate in clinical practice. Although in our cohort, a comprehensive evaluation of tolerance was performed at an age where the child is more stable than in the first year of life, sometimes it is difficult to be aware of over restriction or poor adherence to diet and higher Phe intake. The Guldsberg's phenotype classification adopted for our work classifies PKU forms accurately but leaves HPA definition without a higher boundary. In a milder population like ours, HPA patients are also included in a continuum that goes from those that will never require therapeutic intervention to others that have to be followed carefully and may require diet or even low protein substitutes. Moreover, the regular Argentine diet includes plenty of protein, and even patients with HPA, are at risk of protein overload if the diet is not guided and recommendations are not specified. So, consequently, genotype information would allow establishing individualized treatment rectifying over or under restriction. $[31,32,33]$ 
Several patients in this cohort were tested for response to dihydrochloride $\mathrm{BH}_{4}$, the synthetic cofactor of the enzyme [11]. It is now accepted that a subset of patients would benefit from using this drug.[15,34] As the genotype determines PAH activity and the metabolic phenotype, it is also useful to select patients to be tested or treated. Moreover, the presence of two null mutations makes the testing unnecessary.[11,15]

In Argentina, diet is the first treatment offered to PKU patients. Nevertheless, sapropterin has become available, and $\mathrm{BH} 4$ testing if possible, is performed in the neonatal period mainly because it provides the possibility of assessing $\mathrm{BH} 4$ deficiencies that are difficult to exclude rapidly because of unavailable biochemical studies.[15] In this context, phenotype would complement the information in each patient helping to select the adequate treatment .Thus, though it may seem in some way sophisticated in our medium and still not available for every patient, genotype indeed allows for personalized treatment and follow-up.[32]

Our results highlight the utility of genotype in the provision of appropriate genetic counseling for PKU patients. The report of PAH mutation spectrum benefits the whole PKU community, sharing patients' clinical manifestations and providing useful data to select therapeutic options, and design individualized therapy.Other tools that help to understand better the residual activity of the inherited PAH such as activity landscapes or the measure of isotopic breathed dCO2 would also be useful for the patient's management.[35]

Moreover, recently published evidence on genotype/ phenotype efficient correlation with the GPVs score, would also help in the patient characterization. Unfortunatelly in our cohort, although calculated and provided in the table for each patient, GPV was not used as suggested because the data on pretreatment Phe levels could not be retrieved accurately.[14]

Summing up,we studied a large cohort of PKU Argentine patients to asses their genotype and genotype-phenotype correlation. Our population is highly heterogeneous with milder clinical forms and predominant Mediterranean influence (mainly Spanish), but with differences with other Latin-American countries.

The most frequent pathogenic variants found were p.Arg261Gln, c.1066-11G>A, p.Arg408Trp, p.Tyr414Cys, p.Ala403Val, p.Val388Met, p.ArgR158Gln, p.Leu48Ser, and p.Ile65Thr. $90.3 \%$ of the patients studied were compound heterozygous. Predicted and observed phenotype were overall concordant (62.5\%) except in moderate patients where genotype predicted a milder form than the one seen in the clinical ground. The majority of discordant moderate patients carried the p.Arg261Gln, and p.Val388Met variants. The genotype is a useful tool to manage PKU patients, mainly because of alternative therapies and allowing individualized treatment.

\section{Disclosure}

A. Chiesa is a researcher from the Health Research Council of the Government of the City of Buenos Aires.

\section{Declaration of Conflicting Interests}

The authors declare that there is no conflict of interest.

\section{References}

1. Eisensmith R, Woo S. Molecular basis of phenylketonuria and related hyperphenylalaninemias: mutations and polymorphisms in the human phenylalanine hydroxylase gene. Hum Mutat. 1992;1(1):13-23. doi:10.1002/ humu. 1380010104

2. Scriver CR. The PAH gene, phenylketonuria, and a paradigm shift. Hum Mutat. 2007;28(9):831-845. doi:10.1002/ humu.20526

3. Aldamiz-Echevarria L, Llarena M, Bueno MA, et al. Molecular epidemiology, genotype-phenotype correlation and $\mathrm{BH} 4$ responsiveness in Spanish patients with phenylketonuria. J Hum Genet. 2016;61(8):731744. doi:10.1038/jhg.2016.38

4. Scriver CR, Waters PJ. Monogenic traits are not simple: lessons from phenylketonuria. Trends Genet. 1999;15(7):267-272. doi:10.1016/S0168-9525(99)01761-8

5. Blau N. Genetics of phenylketonuria: then and now. Hum Mutat. 2016;37(6):508-515. doi:10.1002/humu.22980

6. Werner ER, Blau N, Thony B. Tetrahydrobiopterin: biochemistry and patho physiology. Biochem J. 2011;438(3):397-414. doi:10.1042/BJ20110293

7. Specola N, Chiesa A. Alternative therapies in PKU. J Inborn Errors Metab Screen. 2017;5:1-5.doi: $10.1177 / 2326409816685734$

8. Blau N, Longo N. Alternative therapies to address the unmet medical needs of patients with phenylketonuria. Expert Opin Pharmaco. 2015;16(6):791800. doi:10.1517/14656566.2015.1013030

9. Harding CO. New era in treatment for phenylketonuria: pharmacologic therapy with sapropterin dihydrochloride. Biologics. 2010;4:231-236. doi:10.2147/BTT.S3015

10. Camp KM, Parisi MA, Acosta PB, et al. Phenylketonuria scientific review conference: state of the science and future research needs. Mol Genet Metab. 2014;112(2):87122. doi:10.1016/j.ymgme.2014.02.013

11. Blau N, Hennermann JB, Langenbeck U, Lichter-Konecki U. Diagnosis, classification, and genetics of phenylketonuria and tetrahydrobiopterin (BH4) deficiencies. $\mathrm{Mol}$ Genet Metab. 2011;104(suppl):S2-S9. doi:10.1016/j. ymgme.2011.08.017

12. Vishnopolska SA, Turjanski AG, Herrera Piñero M, et al. Genetics and genomic medicine in Argentina. Mol Genet Genomic Med. 2018;6(4):481-491. doi:10.1002/mgg3.455

13. Guldberg P, Rey F, Zschocke J, et al. A European multicenter study of phenylalanine hydroxylase deficiency: classification 
of 105 mutations and a general system for genotype-based prediction of metabolic phenotype. Am J Hum Genet. 1998;63(1):71-79. doi:10.1086/301920

14. Garbade SF, Shen N, Himmelreich N, et al. Allelic phenotype values: a model for genotype-based phenotype prediction in phenylketonuria. Genet Med. 2019;21(3):580590. doi:10.1038/s41436-018-0081-x

15. Muntau AC, Adams DJ, Bélanger-Quintana A, et al. International best practice for the evaluation of responsiveness to sapropterin dihydrochloride in patients with phenylketonuria. Mol Genet Metab. 2019;127(1):111. doi:10.1016/j.ymgme.2019.04.004

16. Zschocke J. Phenylketonuria Mutations in Europe Mutation. Hum Mutat. 2003;21(4):345-356. doi:10.1002/humu.10192

17. Desviat LR, Perez B, Gamez A et al. Genetic and phenotypic aspects of phenylalanine hudroxylase deficiency in Spain: molecular survey by regions. Eur J Hum Genet. 1999;7(3):386-92. doi: 10.1038/sj.ejhg.5200312

18. Daniele A., Cardillo G, Pennino C, et al. Epidemiology of Phenylalanine Hydroxylase Deficiency in Southern Italy: a 96\% Detection Rate with Ten Novel Mutations. Ann Hum Genet. 2007;71(Pt 2):185-193. doi:10.1111/j.14691809.2006.00328.x

19. Santos LL, Castro-Magalhães M, Fonseca CG, et al. PKU in Minas Gerais State, Brazil: mutation analysis. Ann Hum Genet. 2008;72(Pt 6):774-779. doi:10.1111/j.14691809.2008.00476.x

20. Desviat LR, Pérez B, De Lucca M, Cornejo V, Schmidt B, Ugarte M. Evidence in Latin America of recurrence of V388M, a phenylketonuria mutation with high in vitro residual activity. Am J Hum Genet. 1995;57(2):337-342.

21. Hamilton V, Santa María L, Fuenzalida K, et al. Characterization of Phenyalanine Hydroxylase Gene Mutations in Chilean PKU Patients. JIMD Rep. 2018;42:7177. doi:10.1007/8904_2017_85

22. Vela-Amieva M, Abreu-González M, González-del Angel A, et al. Phenylalanine hydroxylase deficiency in Mexico: genotype-phenotype correlations, $\mathrm{BH} 4$ responsiveness and evidence of a founder effect. Clin Genet. 2015;88(1):6267. doi:10.1111/cge.12444

23. Pey AL, Desviat LR, Gámez A, Ugarte M, Pérez B. Phenylketonuria: genotype-phenotype correlations based on expression analysis of structural and functional mutations in PAH. Hum Mutat. 2003;21(4):370-378. doi:10.1002/ humu.10198

24. Trefz FK, Burgard P, König T, et al. Genotype-phenotype correlations in phenylketonuria. Clin Chim Acta. 1993;217(1):15-21. doi:10.1016/0009-8981(93)90233-T

25. Kayaalp E, Treacy E, Waters PJ, Byck S, Nowacki P, Scriver CR. Human phenylalanine hydroxylase mutations and hyperphenylalaninemia phenotypes: a metanalysis of genotype-phenotype correlations. Am J Hum Genet. 1997;61(6):1309-1317. doi:10.1086/301638

26. Trunzo R, Santacroce R, Shen N. In vitro residual activity of phenylalanine hydroxylase variants and correlation with metabolic phenotypes in PKU. Gene. 2016; 594(1):138-143. doi:10.1016/j.gene.2016.09.015

27. van Wegberg AMJ, MacDonald A, Ahring K, et al. The complete European guidelines on phenylketonuria: diagnosis and treatment. Orphanet J Rare Dis. 2017;12(1):162. doi:10.1186/s13023-017-0685-2

28. Leandro P, Rivera I, Lechner MC, de Almeida IT, Konecki D. The V388M mutation results in a kinetic variant form of phenylalanine hydroxylase. Mol Genet Metab. 2000;69(3):204-212. doi:10.1006/mgme.2000.2970

29. Vieira Neto E, Laranjeira F, Quelhas D. Genotypephenotype correlations and $\mathrm{BH} 4$ estimated responsiveness in patients with phenylketonuria from Rio de Janeiro, Southeast Brazil. Mol Genet Genomic Med. 2019;7(5):e610. doi:10.1002/mgg3.610

30. Gámez A, Perez B, Ugarte M, et al Expression analysis of phenylketonuria mutations. Effect on folding and stability of the phenylalanine hydroxylase protein. $J$ Biol Chem. 2000;275(38):29737-29742. doi:10.1074/ jbc.M003231200

31. Singh RH, Cunningham AC, Mofidi S, et al. Updated, web-based nutrition management guideline for PKU: an evidence and consensus based approach. Mol Genet Metab. 2016;118(2):72-83. doi:10.1016/j.ymgme.2016.04.008

32. Giżewska M, MacDonald A, Bélanger-Quintana A, et al. Diagnostic and management practices for phenylketonuria in 19 countries of the South and Eastern European Region: survey results. Eur J Pediatr. 2016;175(2):261272. doi:10.1007/s00431-015-2622-5

33. Greene CL, Longo N. National Institutes of Health (NIH) review of evidence in phenylalanine hydroxylase deficiency (phenylketonuria) and recommendations/guidelines for therapy from the American College of Medical Genetics (ACMG) and Genetics Metabolic Dietitians International (GMDI). Mol Genet Metab. 2014;112(2):8586. doi:10.1016/j.ymgme.2014.03.005

34. Wettstein S, Underhaug J, Perez B, et al. Linking genotypes database with locus-specific database and genotypephenotype correlation in phenylketonuria. Eur J Hum Genet. 2015;23(3):302-309. doi:10.1038/ejhg.2014.114

35. Danecka MK, Woidy M, Zschocke J, Feillet F, Muntau AC, Gersting SW. Mapping the functional landscape of frequent phenylalanine hydroxylase $(\mathrm{PAH})$ genotypes promotes personalised medicine in phenylketonuria. J Med Genet. 2015;52(3):175-185. doi:10.1136/jmedgenet-2014-102621 